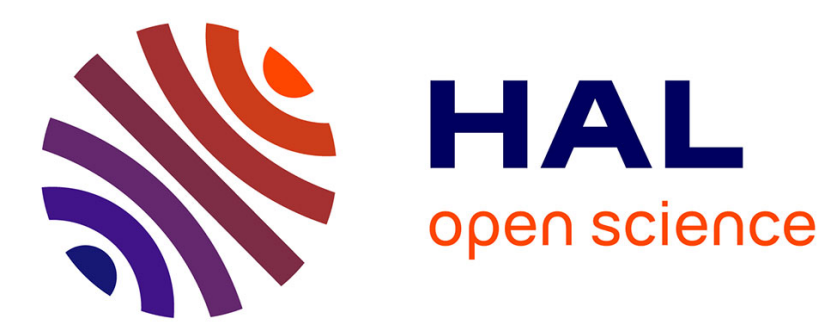

\title{
L'éducation sanitaire par le cinéma dans l'entre-deux-guerres en France \\ Valérie Vignaux
}

\section{To cite this version:}

Valérie Vignaux. L'éducation sanitaire par le cinéma dans l'entre-deux-guerres en France. Sociétés \& Représentations, 2009, Le médecin prescripteur d'images, 28, pp.67-85. 10.3917/sr.028.0067 . halshs01170556

\section{HAL Id: halshs-01170556 https://shs.hal.science/halshs-01170556}

Submitted on 19 Jun 2016

HAL is a multi-disciplinary open access archive for the deposit and dissemination of scientific research documents, whether they are published or not. The documents may come from teaching and research institutions in France or abroad, or from public or private research centers.
L'archive ouverte pluridisciplinaire HAL, est destinée au dépôt et à la diffusion de documents scientifiques de niveau recherche, publiés ou non, émanant des établissements d'enseignement et de recherche français ou étrangers, des laboratoires publics ou privés. 


\section{L'ÉDUCATION SANITAIRE PAR LE CINÉMA DANS L'ENTRE-DEUX-GUERRES EN FRANCE}

Valérie Vignaux

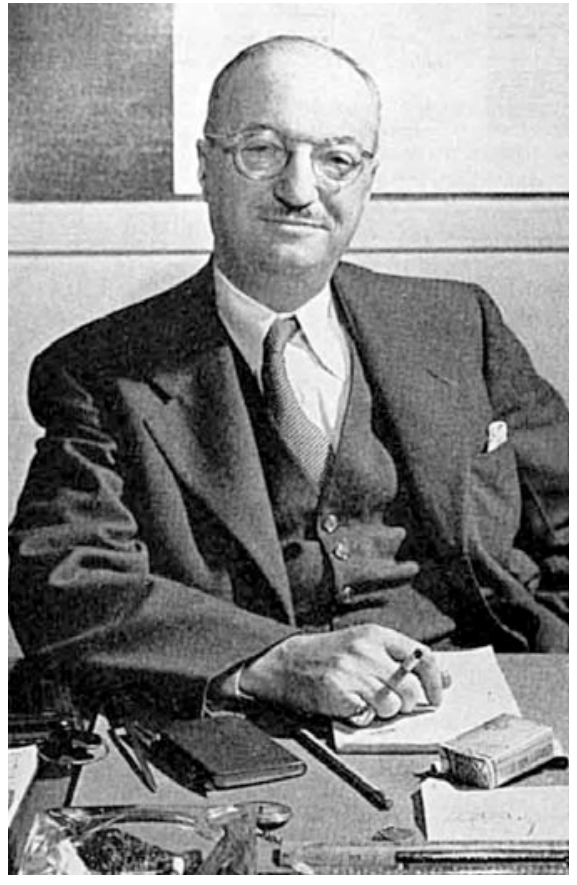

Ill. 1 - Jean Benoit-Lévy, vers 1950.
En 1926, en plaçant sous tutelle publique le service de propagande du Comité de défense contre la tuberculose, le ministère de l'Hygiène sociale reconnaît officiellement l'utilité des activités de prévention par l'image dans la lutte contre les maladies. Une politique ambitieuse de production et de diffusion de films dédiés à la santé publique est alors engagée. Elle conduit en particulier à la mise en œuvre de plusieurs longs-métrages et d'au moins une trentaine de courtsmétrages, dont la réalisation est confiée au cinéaste Jean BenoitLévy $^{1}$, spécialiste reconnu du cinéma éducateur et partenaire privilégié des cinémathèques ministérielles (Agriculture, Santé et Enseignement professionnel). La restitution du

1. Voir Valérie Vignaux, Jean Benoit-Lévy ou le corps comme utopie, une histoire du cinéma éducateur dans l'entre-deux-guerres en France, Paris, AFRHC, 2007, 254 p. 
cadre institutionnel et idéologique légitimant des activités de prévention sanitaire par le cinéma et l'étude des films réalisés par Jean Benoit-Lévy pour la cinémathèque du ministère de la Santé permettront d'évoquer le rôle social réel ou imaginaire dévolu à la médecine dans la France de l'entre-deux-guerres [ill. 1].

\section{LA CINÉMATHÈQUE DE L'HYGIÈNE SOCIALE}

La mise en place d'une cinémathèque dédiée à des questions de santé publique relève des mesures qui, au cours des années Vingt, ont encouragé l'emploi des images à des fins d'instruction ou d'éducation populaire. Dès 1916, une commission extraparlementaire, dite Bessou (du nom de son secrétaire), a été instituée par décret en date du 23 mars afin de "rechercher les meilleurs moyens de généraliser l'utilisation du cinématographe " dans l'enseignement ou l'éducation populaire. Ses conclusions, publiées en $1920^{2}$, ont conduit à la création, la même année, d'un service des films au Musée pédagogique ${ }^{3}$; tandis qu'en 1923 une cinémathèque était officialisée par décret au ministère de l'Agriculture et, en 1925, une cinémathèque de l'Enseignement professionnel s'organisait à la direction de l'Enseignement technique au ministère de l'Instruction publique.

Dernière cinémathèque à être officiellement créée en 1926, la cinémathèque du ministère de la Santé est pourtant celle qui, dans les faits, fut sans doute la première en activité. En effet, dès 1917, face au dépistage d'un nombre considérable de cas de tuberculose par les conseils de révision, le Comité national de défense contre la tuberculose (CNDT), dirigé par Léon Bourgeois ${ }^{4}$, a choisi d'employer, en raison de leur vertu didactique, des images fixes ou animées dans ses campagnes de prévention. Le CNDT, bénéficiant d'une aide matérielle et logistique non négligeable, applique ainsi les méthodes d'une mission venue des États-Unis à l'instigation de la

\footnotetext{
2. Voir Auguste Bessou, Rapport général commission extraparlementaire, chargée d'étudier les moyens de généraliser l'application du cinématographe dans les différentes branches de l'enseignement, ministère de l'Instruction publique et des Beaux-Arts, Paris, Imprimerie nationale, $1920,55 \mathrm{p}$.

3. Le Musée pédagogique a été fondé en 1879 sous la tutelle du ministère de l'Instruction publique pour apporter aux pédagogues les moyens techniques ou intellectuels d'une réflexion sur l'exercice de leur métier.

4. Léon Bourgeois (1851-1925), président du Conseil (1895), ancien ministre de l'Instruction publique et des Beaux-Arts (1898), premier président la Société des Nations (1919-1925), il sera récompensé par le prix Nobel de la paix (1920).
} 
fondation Rockefeller ${ }^{5}$. En 1922, la mission, en prévision de son retour vers les États-Unis, a désigné le CNDT comme son successeur moral et lui a légué un ensemble de dispositifs visuels comprenant, entre autres, un catalogue de films. Un service de propagande est alors instauré sous la tutelle du $\mathrm{D}^{\mathrm{r}}$ Alexandre Bruno, directeur adjoint de la mission Rockefeller, afin de perpétuer les méthodes américaines. Des conférenciers sont formés pour transmettre l'information sanitaire par la parole et la relayer par des brochures ou par la projection de films. Une revue mensuelle, La Vie saine, éditée par le CNDT d'avril 1923 à mai 1933, et dont le tirage passe de 2000 à 15000 exemplaires en 1925, recense les témoignages des spécialistes et, largement illustrée de photos extraites des films commandités à Jean Benoit-Lévy, elle signale aux usagers les dernières productions [ill. 2].

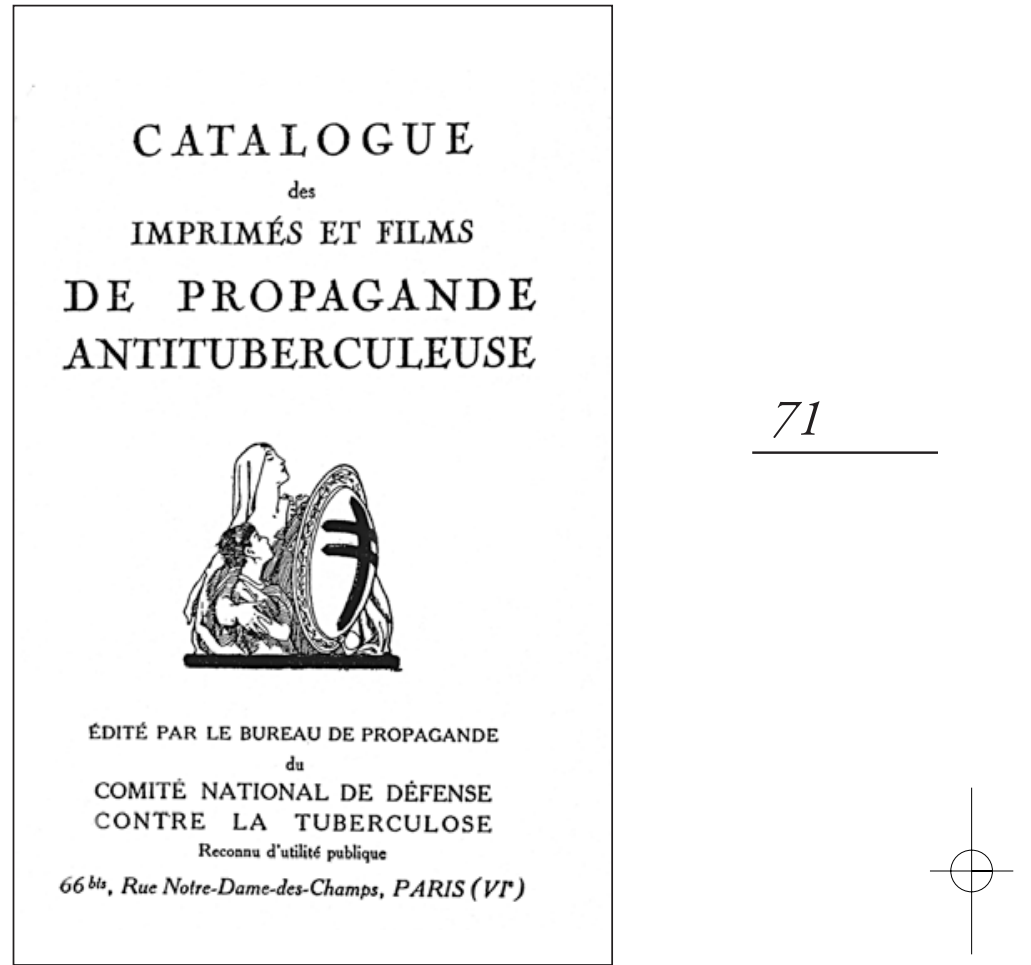

Ill. 2 - Catalogue des imprimés et films de propagande antituberculeuse, édité par le bureau de propagande du Comité national de défense contre la tuberculose, s. d. [Archives du CNDT, Institut Pasteur].

En 1926, à la suite de la création de l'Office national d'hygiène sociale, le service de propagande du CNDT est placé sous tutelle publique. L’Office a été instauré en décembre $1924^{6}$ à l'instigation de Justin Godart ${ }^{7}$,

5. Voir la conférence donnée par le $\mathrm{D}^{\mathrm{r}}$ Williams, directeur de la fondation Rockefeller, au Musée social, le 28 janvier 1922, sous la présidence du professeur Albert Calmette, "La fondation Rockefeller pour la lutte contre la tuberculose en France, son action pendant et depuis la guerre ", Le Musée social, Mémoires et documents, n 2, fév, 1922.

6. Thierry Lefebvre, «Le cinéma contre la syphilis. Pré-enquête sur une lignée cinématographique ", $3^{e}$ Festival CinéMémoire, Paris, CinéMémoire, Cinémathèque française, 1993, pp. 459-462.

7. Voir Annette Wieviorka (dir.), Justin Godart, un homme dans son siècle (1871-1956), Paris, CNRS éd., 2005, 262 p. 
ministre du Travail et de l'Hygiène sociale, pour centraliser sous contrôle public les sources de financement et définir les cadres et modalités de la propagande éducative. Présidé par André Honnorat ${ }^{8}$, il regroupe la plupart des associations, - comme la Ligue nationale contre l'alcoolisme, la Ligue nationale contre le péril vénérien, le Comité national de l'enfance, le Comité central des Croix-Rouges françaises, la Ligue franco-anglo-américaine contre le cancer - qui se préoccupent de santé publique. Ces comités, ligues ou œuvres sont apparus pour la plupart à la fin du XIXe siècle à la suite des découvertes pastoriennes. Les personnalités qui les dirigent ${ }^{9}$ s'attachent à élaborer les cadres idéologiques dans lesquels l'appareil législatif ${ }^{10}$ destiné à améliorer les conditions de vie des classes populaires, victimes et propagatrices des maladies sociales, pourra s'insérer. Ils se réclament d'une science nouvelle, dite " hygiène sociale ", qui trouve dans la contagion microbienne les raisons d'une plus grande solidarité.

Avec son rattachement au service de l'État, la cinémathèque, qui dépendait d'une œuvre privée, voit dans un premier temps le budget qui lui est attribué augmenter. Cependant, lorsqu'en 1935, après la suppression de l'ONHS en 1932, l'État lui retire son aide, la cinémathèque est dans l'obligation de réduire ses activités, son budget annuel chutant soudainement de 600000 à 200000 francs $^{11}$. Décision qui marque à la fois la fin d'une diffusion massive des films d'éducation à l'hygiène auprès des populations urbaines et rurales et l'arrêt d'une production ambitieuse ayant conduit à la réalisation de plusieurs longs-métrages.

\section{LA DIFFUSION DES FILMS}

La cinémathèque prête ses copies aux éducateurs, médecins ou infirmières, qui les utilisent pour accompagner des conférences ou des " causeries ». D’après le catalogue édité par le bureau de propagande, elle emploie

8. André Honnorat, ancien ministre de l'Instruction publique, fut aussi président de la Ligue de l'enseignement, du Comité national de défense contre la tuberculose et président d'honneur de la Ligue nationale française contre le péril vénérien.

9. D’après Jean Benoit-Lévy, regroupés à l'instigation de Justin Godart dans un Parti social de la santé publique, ils militent pour que soit instauré un ministère de la Santé publique. Voir Jean Benoit-Lévy, "L'œuvre sociale de la troisième République » in Henri Laugier (dir.), L’CEuvre de la Troisième République, Montréal, L'Arbre, 1945, pp. 163-187.

10. En 1917, Léon Bourgeois promulgue la loi sur les dispensaires, complétée en 1919 par la loi André Honnorat sur les sanatoriums.

11. Assemblée générale du Comité national en date du 16 mars 1935, sous la présidence de $M$. le $D^{r}$ Queuille, ministre de la Santé publique et de l'éducation physique, Archives du CNDT, cote AS.3 Institut Pasteur. 
également des conférenciers mandatés, indemnisés pour leur intervention et dont les frais de transport sont remboursés. Ils participent à des activités de propagande en lien avec les comités départementaux et leurs actions trouvent place dans des campagnes destinées à recueillir des fonds ${ }^{12}$. Dans les centres urbains, les conférenciers rencontrent les personnes travaillant dans les secteurs de l'" industrie, banque, commerce ", mais aussi les "soldats ». Ils se rendent dans les lycées de jeunes filles pour inciter les élèves à embrasser la carrière d'infirmière visiteuse, et dans les écoles normales d'instituteurs et d'institutrices pour transmettre les prescriptions en matière d'hygiène ${ }^{13}$. Des copies ont été déposées dans les Offices régionaux du cinéma éducateur, présents dans les grandes villes comme Paris, Lille, Lyon, Marseille, etc., et des œuvres postscolaires comme les patronages laïcs peuvent ainsi emprunter ces films gratuitement et les projeter lors de séances payantes, l'argent récolté étant redistribué à la collectivité (réfection des bâtiments ou aide aux nécessiteux).

Lorsqu' elle est le fait des services du CNDT, la propagande " intensive " et méthodique, est organisée en trois " cycles » :

Le 1er cycle a compris pour les enfants et les adultes des causeries sur l'hygiène au foyer. Dans le second, le sujet tuberculose et péril vénérien a été traité pour les adultes ; actuellement nous devons terminer le troisième cycle sur la tuberculose ${ }^{14}$.

Les conférences sont dites "plurivalentes ", car, avec la création de l'Office national d'hygiène sociale, la cinémathèque a reçu en dépôt des films de prévention contre la syphilis. D'après les comptes rendus d'activités établis pour le Conseil de direction par Lucien Viborel, le directeur de la cinémathèque, la diffusion des films ne cesse d'augmenter. En 1923, alors que la mission Rockefeller vient juste de confier la propagande éducative au CNDT, il fait état de 59 conférences employant le cinéma. En 1924, il déclare avoir organisé 246 conférences avec projections, et 639 en 1929. La sollicitation des films est croissante, passant de 400 demandes environ au début des années Vingt, à plus de 1300 en 1930. Le public estimé est important : 103000 personnes en 1924, pour 250000 en 1929 et la circulation importante : 14 départements ont été visités en 1924, 27 en 1927, 42 en 1929, 56 en 1930 et 61 en 1932. Lucien Viborel affirmera qu'en dix ans d'activité (1917-1927) :

12. Catalogue des imprimés et films de propagande antituberculeuse, Bureau de propagande du Comité national de défense contre la tuberculose, s. l. n. d., p. IV.

13. Voir Lucien Viborel, «Éducation populaire et cinématographe : c'est le plus sûr auxiliaire de l'enseignement de l'hygiène ", La Vie saine, n 44, mars 1927.

14. Conseil de direction du Comité national, séance du 15 novembre 1924, Archives du CNDT, cote AS.1. Institut Pasteur. 
L'ORGANISATION DES CENTRES RÉGIONAUX DE PROPAGANDE AVEC GROUPES AUTOMOBILES EFFECTUÉE EN FRANCE PAR L'OFFICE NATIONAL D'HYGIENE SOCIALE.

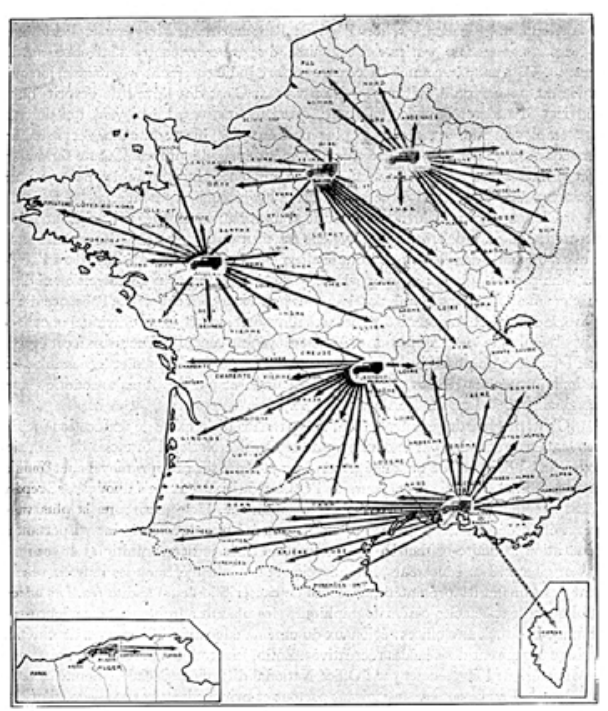

Ill. 3 - L'organisation des groupes automobiles, Revue internationale du cinéma éducateur, 1932.
60 départements ont été méthodiquement visités. Plus de 10 millions de personnes ont été atteintes par notre propagande dont les principaux moyens étaient les équipes volantes, créées par nos amis de la mission Rockefeller. Chaque équipe comprenait : un conférencier, une conférencière s'adressant aux milieux féminins et traitant de l'hygiène du foyer, un chauffeur-opérateur. Ce personnel était transporté par un camion-automobile muni d'une installation cinématographique et d'un groupe électrogène, ainsi que d'une collection de films variés. [...] 200 films sont couramment projetés. Plus d'un million de séances ont été organisées ${ }^{15}$.

Le premier groupe automobile de propagande par le cinématographe a été organisé en juin 1928 [ill. 3], pour donner dans « le village reculé, le chantier ou la mine éloignés de tout centre ", des présentations de films commentés par la conférence. Une exposition ambulante est annexée à la camionnette et, en 1931, le Comité emploie neuf groupes régionaux automobiles, dont deux circulent en Afrique du Nord, financés à part égale par le Comité national de défense contre la tuberculose et l'Office national d'hygiène sociale. Le dispositif est apprécié mais coûteux :

Ce genre d'activités ne peut être assuré, au moins actuellement, qu'avec l'aide d'importantes subventions. La séance cinématographique ambulante ne fait pas encore recette suffisante, et, d'autre part, son fonctionnement est très onéreux ${ }^{16}$

Réduit à deux groupes automobiles en 1934, il cesse de fonctionner en 1935 du fait d'un moindre investissement financier de l'État, et non parce que les séances d'éducation à l'hygiène auraient rebuté les populations, au contraire. Lucien Viborel pouvait, en effet, déclarer en 1924 :

15. Lucien Viborel, «Éducation populaire et cinématographe : c'est le plus sûr auxiliaire de l'enseignement de l'hygiène ", loc. cit.

16. Ibid.

V. Vignaux, "L'éducation sanitaire par le cinéma...” ", S. \& R., n² 28, sept. 2009, pp. 71-87. 
[... partout ces causeries ont été l'objet d'un gros succès - il n'est pas une ville qui ne réclame, dans les divers départements, le renouvellement d'une action semblable à celle qui vient de se dérouler ${ }^{17}$.

\section{DES FILMS D'HYGIÈNE SOCIALE}

La cinémathèque du ministère de l'Hygiène sociale déclare posséder, en 1927, un catalogue de 200 films, augmenté à 500 en $1930^{18}$. D'après les commentaires de Lucien Viborel, ce catalogue est composé des films américains légués par la commission Rockefeller ${ }^{19}$; des titres acquis auprès de Pathé, comprenant les réalisations du $\mathrm{D}^{\mathrm{r}}$ Jean Comandon et les dessins animés d'O'Galop ; des sujets d'enseignement établis par les établissements Gaumont pour illustrer des leçons d'hygiène prodiguées en classe (écoles primaires, enseignement secondaire et supérieur) et des réalisations de Jean Benoit-Lévy :

Parmi tous les louables efforts accomplis dans le domaine de l'enseignement de l'hygiène sociale par le cinématographe, se détache avec un grand relief l'effort de l'Édition française cinématographique, dont le créateur et l’animateur est Jean Benoit-Lévy ${ }^{20}$.

Les films de Jean Benoit-Lévy se distinguent des précédents parce qu'ils ont été réalisés en suivant les prescriptions des services de propagande, ceux du CNDT ou de l'ONHS, et les génériques portent systématiquement mention de l'un ou l'autre organisme. Parmi cet ensemble important, puisque composé de trois longs-métrages et d'au moins une trentaine de courts-métrages, on peut différencier les sujets relevant d'un traitement documentaire et ceux dont le récit repose sur des ressorts fictionnels.

Les documentaires d'une vingtaine de minutes décrivant les institutions sanitaires sont les films les plus anciens. On découvre, par exemple, dans Un village sanatorium (1924), le $\mathrm{D}^{\mathrm{r}}$ Alexandre Bruno, directeur adjoint de

17. Conseil de direction du Comité national, séance du 15 novembre 1924. Archives du CNDT, cote AS.1. Institut Pasteur.

18. Lucien Viborel, "Éducation populaire et cinématographe... ", loc. cit. ; Lucien Viborel, "Le cinéma créateur d'impressions distrayantes est, avant tout, un prodigieux agent d'éducation ", Revue internationale du cinéma éducateur, n 5, mai 1930.

19. Voir Thierry Lefebvre, "Les films diffusés par la mission américaine de prévention contre la tuberculose (Mission Rockefeller, 1917-1922) ", 1895, n ${ }^{\circ} 11$, déc. 1991, pp. 101-106.

20. Marcel Colin, "Le film d'éducation et d'hygiène ", La Cinématographie française, 24 déc. 1927. 
la mission Rockefeller, inaugurant un terrain. Les établissements présentés sont majoritairement réservés aux femmes : Sanatorium de la Tuyolle (vers 1925), Établissement du Glandier à Beyssac (vers 1925) ; ou aux enfants : Préventorium Lannelongue à Oléron (vers 1925), École de plein air de Pantin (vers 1925), École en plein air du XIX arrondissement (vers 1925). Seul le Sanatorium de Bligny (vers 1925), sous tutelle militaire, est destiné aux hommes. La présentation suit un protocole identique de film en film : description des bâtiments, de l'encadrement médical et des activités curatives associées à l'air et au soleil. Films de propagande, ils ne montrent que très rarement la maladie et privilégient les pratiques hygiénistes : exercices en plein air, cures de soleil et examens médicaux. Ils valorisent la présence des médecins directeurs, véritables figures patriarcales qui veillent sur le rétablissement des malades, et vantent l'encadrement scolaire et éducatif. Projetés au cours de causeries, ils doivent convaincre les adultes du bienfondé des traitements et inciter les parents à se séparer de leurs enfants. Documents rares, ils constituent très probablement les seules images documentaires enregistrées dans les établissements médicaux, à l'aube des années Vingt [ill. 4].

\section{OFFICE PUBLIC DHYGIËNE SOCIALE DU DEPXRIEIERT DE LA SEME 9, Place de PHôtel de Ville PARIS IV Service de la Propagande}
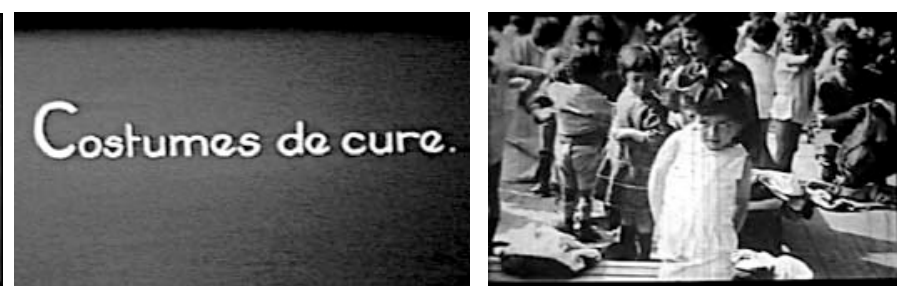

Ill. 4 - École de plein air de Pantin, réalisation Jean Benoit-Lévy, 1929.

Certaines des images documentaires réapparaissent à des fins d'illustration dans les films de "statistiques " comme L'Armement anti-vénérien (vers 1928) ou L'Armement anti-tuberculeux (vers 1928) ; films qui reposent presque en totalité sur des intertitres où abondent les chiffres, appuyés par des schémas animés. Probablement projetés lors de présentations officielles, ils semblent avoir été conçus pour convaincre d'éventuels donateurs ou pour rassurer les édiles sur l'emploi fait des subventions publiques.

Dans cet ensemble commandité par la cinémathèque du ministère de la Santé, les films de fiction sont prépondérants. De forme plus attrayante, comparables aux spectacles cinématographiques de divertissement, les fictions sont censées faciliter l'adhésion des spectateurs et ainsi transmettre 
largement les prescriptions en matière d'éducation sanitaire. Les très courtes fictions de trois minutes environ comme La Contagion par les crachats, La Contagion par les jouets portés à la bouche, La Bonne voisine ou L'Apéritif, réalisées aux alentours de 1925, sont centrées sur un message simple et univoque. L'hygiène est une responsabilité maternelle ; et il revient à la mère de transformer la misère en richesse, en insufflant par la propreté et la santé, dans ces familles monoparentales où les hommes sont défaillants ou absents (car morts à la guerre), comme une promesse de bonheur pour les générations futures. Ordre et propreté sont synonymes de santé et d'élévation sociale par l'éducation. Films très courts, financés pour les trois premiers, d'après les génériques, par la vente du timbre antituberculeux, ils étaient projetés ensemble ou avec d'autres dans le cadre d'un programme.

La présence dans cet ensemble de trois longs-métrages produits et réalisés de 1925 à 1927, La Future Maman, Le Voile sacré et Il était une fois trois amis, témoigne des facilités financières dont a bénéficié, dans un premier temps, la cinémathèque du ministère de la Santé. En effet, les films de fiction ont des budgets élevés, car ils emploient des comédiens, supposent une quantité importante de pellicules (scènes rejouées) et sollicitent du personnel occupé à la lumière, aux décors ou aux costumes. La Future maman (1925), premier des longs-métrages réalisés par Jean Benoit-Lévy, a été écrit par le $\mathrm{D}^{\mathrm{r}}$ Louis-Jules Devraigne ${ }^{21}$, obstétricien et conférencier réputé, opérant dans les Universités populaires depuis 1903 [ill. 5]. Le récit et sa mise en image sont extrêmement simples et entièrement organisés autour du contenu éducatif. Sept leçons (de la préparation de la layette et du bain, à la consultation des nourrissons) sont déclinées successivement et séparées par des intertitres reprenant des adages attribués aux sommités du monde médical : "La mère a couvé l'enfant qui vient de naître : que la chaleur du foyer continue son œuvre ! "; ou encore : "Le cœur et le lait d'une mère ne se remplacent jamais " (attribué au Pr Pinard), etc. L'ensemble est dramatisé en opposant Margot, élève assidue d'une école de puériculture, à Mabu, voisine d'un certain âge, aux " préjugés séculaires » [ill. 6]. Leur relation est l'occasion de découvrir des images documentaires filmées dans un dispensaire et les cours auxquels assiste Margot, prodigués par une institutrice employant le cinématographe. Le film pourrait avoir été réalisé pour illustrer les cours de puériculture qui, depuis 1924, sont aux programmes

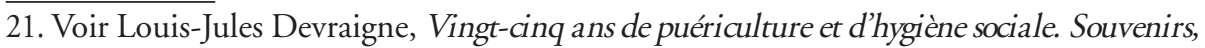
conférences, films, Paris, Gaston Doin et $\mathrm{C}^{\text {ie }}$ éd., 1928, 342 p. 


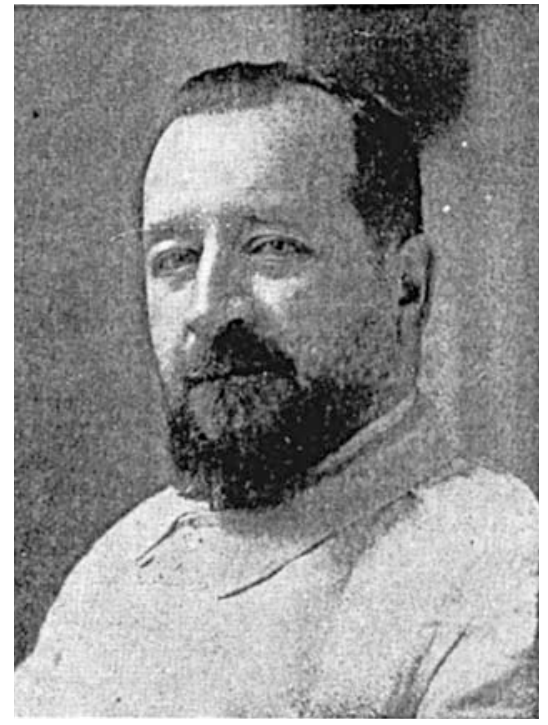

Ill. 5 - M. le Dr Devraigne, chef de la maternité à l'hôpital Lariboisière, vers 1925.

des écoles primaires pour filles. Il semble cependant que les prescripteurs souhaitaient s'adresser prioritairement aux femmes adultes :

Le fameux film intitulé la Future maman, réalisé par le Dr Devraigne et Jean Benoit-Lévy, a porté aux ouvrières des usines et jusqu'au fond de nos villages les élémentaires notions de puériculture dont l'ignorance est bien souvent une cause de plus à la mortalité infantile ${ }^{22}$.

Le Voile sacré (1926) reprend les éléments dramatiques mis en place par $\mathrm{La}$ Future maman et l'on retrouve Mabu, la villageoise, alors qu'elle aide Margot, sa jeune protégée, à ouvrir un dispensaire à la campagne. Margot, qui assiste le médecin dans sa tâche, traverse la campagne à bicyclette pour secourir les âmes en peine : son visage, sublimé par le gros plan en déplacement, apparaît en surimpression avec les paysages. Elle convainc les parents de Pierre, qui vient de décéder d'une tuberculose attrapée en ville, de transformer leur ferme en fondation pour les " tout-petits ». Elle empêche le suicide de Marie, fille de ferme engrossée par son patron, et incite à la création d'une maternité. Le dispensaire jouxte l'école où l'instituteur a fondé un foyer social. "Deux ans plus tard ", Margot a épousé l'instituteur, et le ministre de la Santé venu visiter leur centre d’hygiène, la décore, ainsi que Mabu, de la médaille de l'Assistance publique. La représentation, qui fait l'apologie de ce nouveau métier féminin, est conforme aux discours propagandistes. Lucien Viborel dans La Vie saine en décembre 1928, écrivait :

Elle [l'infirmière visiteuse] vient, sous le signe de la solidarité sociale, "servir » dans toute la noblesse du mot, le malade, et en ce malade "l'humanité souffrante » et elle sait adapter les conseils et les notions au milieu où elle exerce sa haute mission et au cas précis qui l'occupe.

Alors qu'il réalise un long-métrage à l'instigation de la Ligue contre le péril vénérien, Jean Benoit-Lévy inscrit à nouveau son récit dans une propagande nataliste et se félicite que son film ait pu être projeté dans les

22. (Anonyme), "Le cinéma éducateur : la grande puissance éducative du film doit servir à "élever" le peuple tout en le distrayant ", La Solidarité, 11 oct. 1936. 

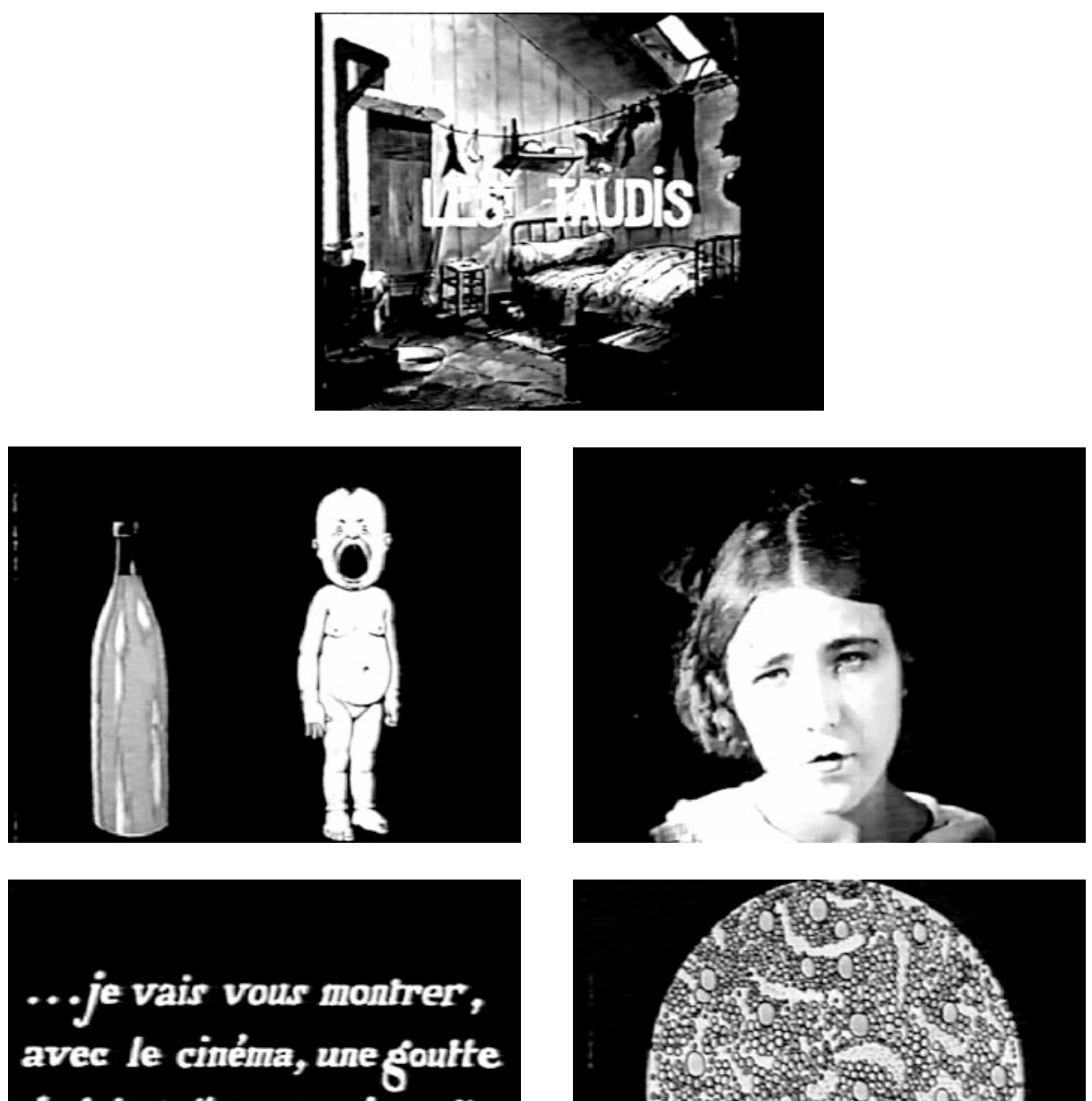
de lait telle qu'on la voit au micrascope.

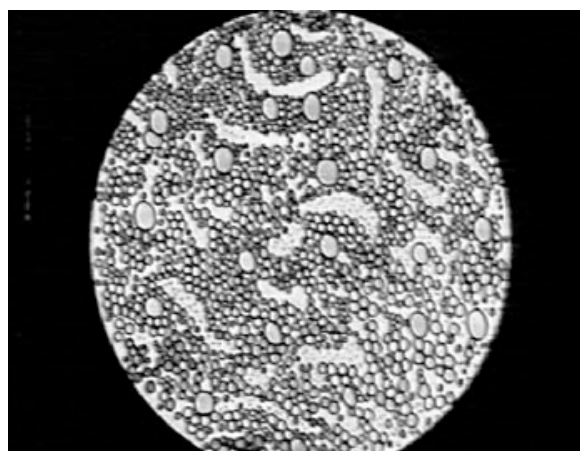

\section{Ma chère petite,}

décidément tu as comverti la vieille radoteuse que $j$ 'érais! $C$ 'est beau I'instruction!!!

"La mére a couvé I'enfant qui vient de naitre: que la chaleur du foyer continue son ceuvre!" Dr. DEMELIN, ex-Rofesseur à la Moternité de Paris.

Ill. 6 - La Future maman, scénario du Dr Devraigne, réalisation Jean Benoit-Lévy, 1925. 

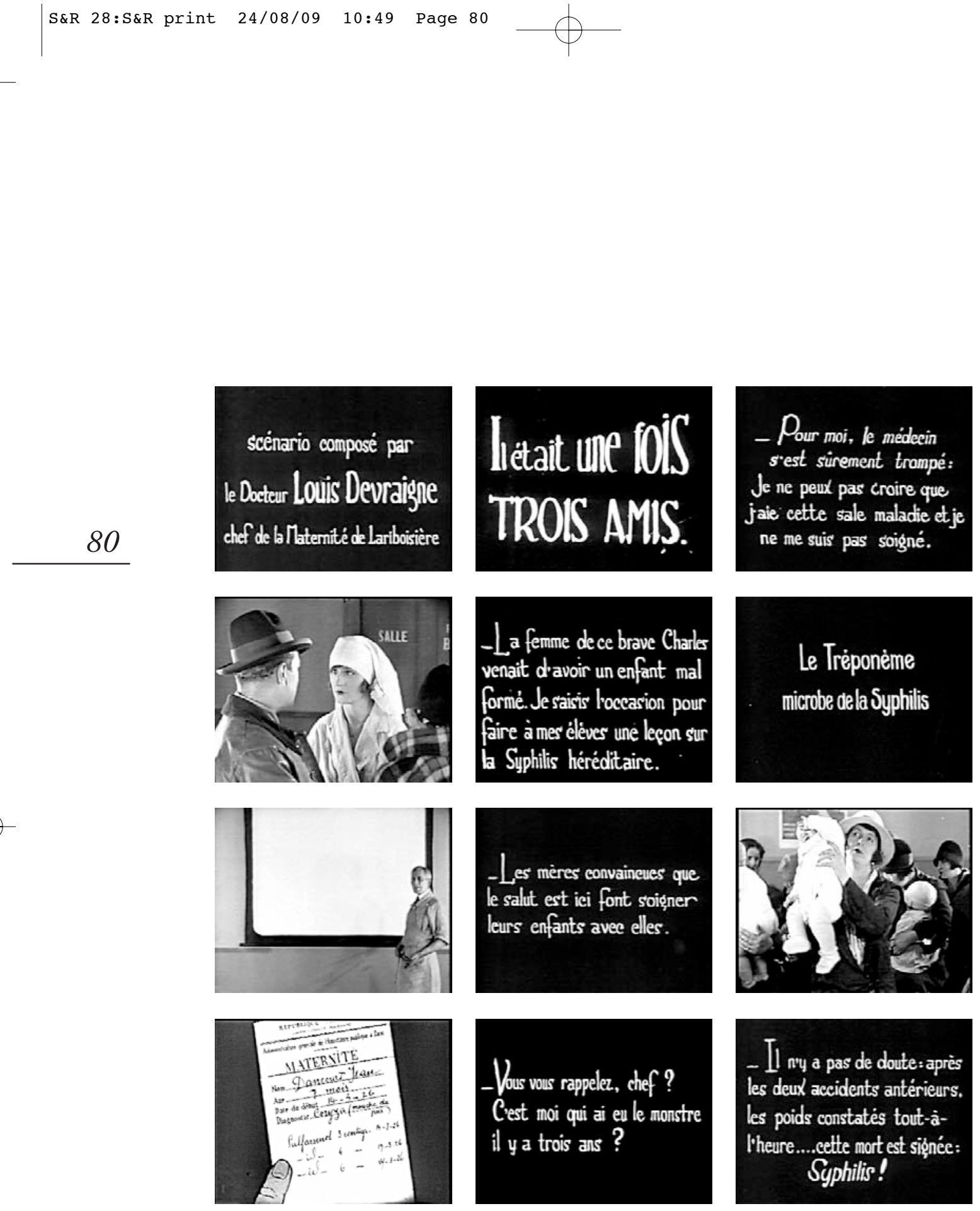

Ill. 7 - Il était une fois trois amis, scénario du Dr Devraigne, réalisation Jean Benoit-Lévy, 1927. 
écoles de jeunes filles ${ }^{23}$. Il était une fois trois amis (1927), écrit par le $\mathrm{D}^{\mathrm{r}}$ Devraigne envisage la maladie en tant que cause de mortalité infantile [ill. 7]. Un médecin raconte à son fils une histoire empruntée à sa carrière : trois amis, Charles, garagiste et ancien chauffeur du médecin, Georges, employé de banque, et Jacques, cultivateur, se retrouvent pour fêter leurs fiançailles. Georges s'est fait soigner pour une syphilis contractée un soir de bal au régiment, alors que les deux autres pensent avoir échappé à la maladie. "Vingt mois " plus tard, les trois ménages se retrouvent pour le baptême du second fils de Georges. La femme de Jacques est absente pour cause de fausse couche, et celle de Charles est à la maternité où elle donne naissance à un enfant mal formé, un "monstre ». Le médecin convoque son ancien chauffeur et lui apprend qu'il est porteur d'une " hérédo seconde ", car la syphilis lui aurait été transmise par son père. Il l'incite à venir à la consultation pour découvrir les modalités de dépistage et de soin. "Cinq ans " plus tard, les trois amis sont réunis pour la naissance du premier enfant de Jacques. Malheureusement, le bébé décède, car il est lui aussi porteur de cette "affreuse maladie ». Jacques, prenant enfin conscience de son mal, accepte de se faire soigner. "Vingt-cinq ans " après, ils se retrouvent pour fêter leurs noces d'argent et un photographe s'évertue à rassembler dans le cadre les trop nombreuses descendances. Le curé et l'instituteur ont été conviés aux réjouissances et le premier se désole : "Quelle jolie fête, M. l'Instituteur. Et quel dommage que le bon docteur n'ait pu assister au triomphe de son art! ». Le film s'achève alors que le médecin enjoint son fils à transmettre à ses amis le message : "Dis-leur qu'il n'y a pas de maladies honteuses mais seulement des malades honteux. "

Ces films à la facture ambitieuse s'attachent à informer les classes populaires en les plaçant dans un cadre paternaliste et rassurant. Les spectateurs sont invités à suivre les aventures de personnages familiers, pensés à leurs images, qu'ils retrouvent de film en film. On reconnaît en effet, dans le rôle de la mère de Jacques, dans Il était une fois trois amis, la comédienne qui interprète Mabu dans La Future maman, également présente dans Le Voile sacré. Le dispositif éducateur est également souligné et nous assistons dans

23. "Un film comme Il était une fois trois amis sur la syphilis, n’a peut-être pas joui de la publicité plus ou moins malsaine de certaines autres bandes... spectaculaires. Mais j'ai la joie et l'orgueil de déclarer qu'il a passé dans les lycées de jeunes filles. " Jean Benoit-Lévy, "Une grande manifestation de cinéma culturel et éducatif », Le Petit niçois, 4 nov. 1932. Il était une fois trois amis a été présenté à la Sorbonne « devant 3000 personnes, alors que 2000 au moins ne pouvaient entrer ". Louis-Jules Devraigne et Jean Benoit-Lévy "Le cinéma éducateur » in Vingt-cinq ans de puériculture et d'hygiène sociale, op. cit., p. 336. 
La Future maman, Le Voile sacré ou Il était une fois trois amis à une séance d'information illustrée par le cinéma. Or, qu'il s'agisse des documentaires ou des films de fiction, la place des personnages féminins est prépondérante. Taudis, tuberculose, syphilis et alcoolisme sont envisagés pour responsabiliser les femmes et faire d'elles les garantes de la bonne santé de la nation. Public privilégié par ces films d'éducation sanitaire, leur émancipation sociale par le travail apparaît comme une importante mutation sociale et culturelle, car manifestement elle préoccupe les politiques qui se font les chantres des enjeux natalistes.

Au début des années Trente, l'activité de la cinémathèque connaît un très net ralentissement. Le désengagement financier de l'État coïncide en effet avec l'arrivée du cinéma parlant, qui recouvre la parole du conférencier et oblige de plus à renouveler un matériel coûteux rendu obsolète. La progression de la scolarisation conjuguée aux actions de prévention orchestrées dans le sillage d'une médecine sociale, ont par ailleurs contribué à une baisse notable de la morbidité. De plus, les modèles de transmission des connaissances, issus de la vulgarisation scientifique et qui légitimaient le recours à la fiction pour ces films d'éducation populaire, sont dorénavant désavoués par les prescripteurs, déclarés comme faisant obstacle à la recherche scientifique. L'œuvre de Jean Benoit-Lévy, issue de la commande publique et destinée à des fins d'éducation sanitaire, subit ces réorientations à la fois techniques, idéologiques et économiques. En 1930, nommé secrétaire du Comité d'études médico-chirurgicales par le cinématographe $e^{24}$, " honoré des patronages des ministères de l'Éducation nationale et des Affaires étrangères ${ }^{25}$ ", il réalise, sous la tutelle des professeurs Antoine Gosset, Charles Laubry, Roger Leroux, Gustave Roussy et Léon Bernard, des films destinés à illustrer des cours d'enseignement de la chirurgie. D'après la plaquette de présentation, le Comité a pour but le progrès des méthodes de recherches et d'enseignement biologique par le cinéma, et plus spécialement dans le domaine médico-chirurgical ; l'amélioration à ces fins de la technique cinématographique; l'organisation de la collaboration et des échanges intellectuels; la réalisation de films (muets puis sonores) et leur diffusion dans les milieux intéressés [ill. 8]. Le centre de recherche est composé de deux laboratoires techniques : le premier à Villejuif se consacre aux recherches biologiques et d'anatomie et le second,

24. Voir Valérie Vignaux, "Contribution à une histoire de l'enseignement de la chirurgie par le cinéma ", 1895, n 44, déc. 2004, pp. 73-86.

25. Jean Benoit-Lévy, Les Grandes Missions du cinéma, Montréal, Lucien Parizeau et Cie, 1945 , p. 67. 


\section{Le Comité français} d'Études

\section{médico - chirurgicales par le Cinématographe}

\section{Comité de direction : \\ Président :}

M. G. ROUSSY, Doyen de la Faculté de Médecine, Membre de l'Académie de Médecine.

Vice-présidents :

M. A. GOSSET, Professeur à la Faculté de Médecine, Membre de l'Institut.

M. Ch. LAUBRY, Médecin des Hôpitaux, Membre de l'Académie de Médecine.

Secrétaire général :

M. Roger LEROUX, Professeur agrégé à la Faculté de Médecine.

Secrétaire :

M. Jean BENOIT-LÉVY.

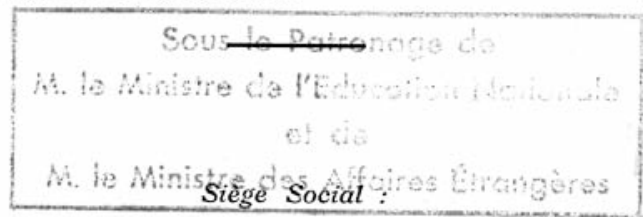

FACULTÉ DE MÉDECINE

21, Rue de l'École-de-Médecine, PARIS

Ill. 8 - Le Comité français d'études médico-chirurgicales par le cinématographe, vers 1930 . 
dans le service du Pr Gosset à l'hôpital de la Salpêtrière, à la chirurgie. L'activité du Comité est importante, malgré les difficultés matérielles, et perdure tout au long de l'entre-deux-guerres :

Au moment de la déclaration de guerre en 1939, le Comité était en plein fonctionnement. Nous avions dans nos laboratoires les appareils nécessaires dont certains avaient été fabriqués spécialement suivant les données produites par nos expériences. À l'hôpital de la Salpêtrière, une salle d'opération était spécialement équipée. Des savants vinrent un peu partout profiter de notre expérience et de nos installations. [...] Notre Comité devint ainsi un véritable centre de recherches, un foyer de travail, un pôle d'attraction ${ }^{26}$.

Toutefois, malgré la diminution puis la disparition de la commande publique, Jean Benoit-Lévy s'efforcera, dans le cadre de ses films de fiction destinés aux circuits commerciaux, de perpétuer les idéaux qui régissaient ses personnages empruntés au monde médical. Itto (1935), par exemple, légitime la présence française au Maroc en tant qu'elle contribue à la diffusion des progrès induits par la connaissance scientifique. Un médecin, parce qu'il a guéri un troupeau de moutons malades, obtient le ralliement d'une tribu à la nation française. Et, grâce à la vaccination, il parvient à sauver les enfants qu'un charlatan mettait en danger. Hélène (1936), réalisé l'année suivante, présente une jeune femme résolue à passer son doctorat de médecine malgré le fait qu'elle soit sans soutien de famille. Un professeur, pourtant peu favorable au travail des femmes, l'accepte comme élève et l'emploie au laboratoire. Devenue mère célibataire car son compagnon s'est suicidé, elle parvient à convaincre le professeur qui, de son côté, a été rejeté par sa femme chanteuse, de ne pas abandonner son travail, trouvant dans la recherche scientifique un réconfort mutuel.

Dans l'entre-deux-guerres, les politiques sollicitèrent le cinéma et les conférenciers parce qu'ils souhaitaient transmettre massivement les réformes mises en place, réformes qui visaient à modifier en profondeur les mentalités et les usages des classes populaires. Cependant, les commanditaires de ces films ignoraient qu'en recourant aux représentations pour légitimer leur action, en s'appliquant à les définir pour mieux les maîtriser et les diffuser, elles les obligeraient à se renouveler s'ils ne souhaitaient pas disparaître au rythme avancé des mutations sociales ou techniques. Les films

26. Ibid., p. 70.

V. Vignaux, "L'éducation sanitaire par le cinéma...”", S. \& R., n 28, sept. 2009, pp. 71-87. 
de propagande nataliste, par exemple, en vantant la mise en place des structures (crèches, maternités, écoles maternelles) qui permettaient aux femmes de concilier la maternité avec des activités rémunérées, les aidèrent à s'émanciper de la seule sphère familiale.

Ainsi la restitution des représentations du monde médical, telles que produites et diffusées par les instances publiques, donne-t-elle à voir les transformations idéologiques mais aussi épistémologiques qui assaillent la société française dans l'entre-deux-guerres. 